\title{
Conductivity studies of graphene oxide on biopolymer electrolyte
}

\author{
Syakirah Shahrudin ${ }^{1}$, Azizah Hanom Ahmad 1, 2,* \\ ${ }^{1}$ Faculty of Applied Sciences, Universiti Teknologi MARA (UiTM), 40450 Shah Alam, Selangor, Malaysia \\ 2Institute of Science, Universiti Teknologi Mara (UITM), 40450 Shah Alam, Selangor, Malaysia
}

\section{A R T I C L E IN F O}

\section{Article history:}

Received 8 August 2016

Received in revised form

21 September 2016

Accepted 7 October 2016

\section{Keywords:}

Biopolymer electrolyte

Cornstarch/NaI

GO

\begin{abstract}
A B S T R A C T
In this research, biopolymer electrolyte has been prepared by doping cornstarch with sodium iodide (NaI) using solution casting method. The incorporation of $25 \% \mathrm{NaI}$ resulted in maximum conductivity of the electrolyte at $1.46 \times 10^{-4} \mathrm{~S} \mathrm{~cm}^{-1}$. The enhancement of the electrical conductivity to $2.61 \times 10^{-3} \mathrm{~S} \mathrm{~cm}^{-1}$ was achieved with further addition of graphene oxide (GO). The effect of GO on the electrolyte system were investigated by deploying electrical impedance spectroscopy (EIS) measurement at temperature of $298 \mathrm{~K}-373 \mathrm{~K}$. Electrical conductivity as function of temperature have been investigated and revealed that the conductivity decreases as temperature increase. Dielectric studies were performed in order to study the ion mobility in the electrolyte system.
\end{abstract}

(C) 2016 The Authors. Published by IASE. This is an open access article under the CC BY-NC-ND license (http://creativecommons.org/licenses/by-nc-nd/4.0/).

\section{Introduction}

Polymer electrolytes have received great attention due to their potential uses in solid state electrochemical devices. In the past few years, many natural polymers have been studied as polymer hosts in electrolytes due to cost effective and its environmentally friendly characteristics (Kumar et al., 2012; Shanti, 2011). Starch is one of the natural polymer which is non-toxic, biodegradable and available in abundance (Yusof et al., 2014). The improvement of physical properties of the starch can be obtained by modification with other agents or additives (Syzdek et al., 2010).

Incorporating appropriate salts into the host polymer matrix provides charge carriers to the polymer matrix in order to increase the ionic conductivity of the system. Research in sodium ion polymer electrolyte has been increasing in recent years. A few attempts have tried polymer electrolytes based on sodium ion complexed films (Dzulkurnain et al., 2015). The main advantage of using sodium metal ion is its availability in abundance at a cheaper cost than lithium. Furthermore, softness of the material makes it easier to achieve good contact with other components in the battery (Bhargav et al., 2007).

\footnotetext{
* Corresponding Author.

Email Address: azizahanom@salam.uitm.edu.my (A. H. Ahmad)

https://doi.org/10.21833/ijaas.2016.10.003

2313-626X/C 2016 The Authors. Published by IASE.

This is an open access article under the CC BY-NC-ND license

(http://creativecommons.org/licenses/by-nc-nd/4.0/)
}

Besides that, it has been suggested that by modification of starch with other agent or additives can improve its physical properties. Graphene oxide (GO) are two dimensional carbon materials that have attracted great interest due to high surface area, good electrochemical and mechanical properties $\mathrm{CH}$ et al., 2014; Yu et al., 2012). GO has been found to be more compatible with polymer and it gives advantages to the polymer/GO composites (Dikin et al., 2007; Diwan et al., 2012).

GO, which exhibits many oxygen functional groups on its basal planes and edges, is highly dispersible in water and thus make it a promising material for composites with polymer. The presence of various oxygen containing functional groups with GO moiety leads to synthesis of various composites to improve the desired properties (Latif et al., 2012; Yu et al., 2012; Joshi and Deshmukh, 2016).

Polymer/GO composites attracting more attention because it shows high performance in mechanical and electrical properties much better than any other filler such clay or even nanotubes (Balog et al., 2010; Quan et al., 2009). However, research is still necessary to explore the potential of the conducting polymer graphene composites as solid electrolyte for electrochemical devices.

In the present work, a solid biopolymer electrolyte based on cornstarch/NaI with different amount of GO was investigated. Cornstarch/NaI/GO composites were characterized by electrochemical impedance spectroscopy (EIS) at room temperature and elevated temperature to study the electrical and 
transport behavior of the charge carrier in the electrolyte.

\section{Experiments}

\subsection{Materials}

Cornstarch $\left(\mathrm{C}_{6} \mathrm{H}_{10} \mathrm{O}_{5}\right.$, CAS =9005-25-8, SigmaAldrich) in powder form, Sodium iodide (NaI, MW = 149.89 g/mol, CAS = 7681-82-5, Sigma-Aldrich) and graphene oxide (G0 powder, 15- 20 sheets, SigmaAldrich)

\subsection{Preparation of cornstarch/NaI-GO electrolyte}

In this work the optimum composition of the host polymer electrolyte Cornstarch/NaI obtained is 65:25. GO was added to the host polymer at different percentage and was continuously stirred for few hours in order to achieve a homogeneous mixture. The electrolytes were prepared via solution casting technique using distilled water as the solvent and free standing polymer electrolyte films were obtained.

\subsection{Characterization technique}

The samples were characterized using HIOKI 3531-01 LCR Hi-tester between the frequency ranges of $50 \mathrm{~Hz}$ to $1 \mathrm{MHz}$ at room temperature and at various temperatures $(287 \mathrm{~K}-373 \mathrm{~K})$ were performed.

\section{Results and discussion}

In this work, all samples were analyzed at constant humidity and temperatures ranging from $25^{\circ} \mathrm{C}$ to $90^{\circ} \mathrm{C}$. Samples were placed on a sample holder with the diameter of $2.4 \mathrm{~cm}$. The conductivities of cornstarch biopolymer electrolyte film with various weight percent of $\mathrm{NaI}$ were investigated. The electrical conductivity of the samples were calculated by substitute the value of bulk resistance, $R_{b}$, thickness, $t$ and contact area of each samples into following equation,

conductivity,

$$
\sigma=\frac{\mathrm{t}}{\mathrm{AxR_{ \textrm {b } }}}
$$

where,

$$
\begin{aligned}
& t=\text { thickness of sample }(\mathrm{cm}) \\
& A=\text { contact area of the sample }\left(\mathrm{cm}^{2}\right) \\
& R_{b}=\text { bulk resistance of the sample }(\mathrm{s})
\end{aligned}
$$

Fig. 1 shows the impedance plots of CornstarchNaI electrolytes with a different amount (wt.\%) of $\mathrm{NaI}$. The plots show the intercept over the x-axis at low frequency region represents the bulk resistivity, $\mathrm{R}_{\mathrm{b}}$ of the sample which was used to determine the value of electrical conductivity, $\sigma$ of the electrolyte.
The inclination and bulk resistance of the samples were different from each other due to different ionic mobility and availability of free ions in the system.

The variation of conductivity with salt content is shown in Fig. 2. It is observed that the ionic conductivity cornstarch increases with the addition of $\mathrm{NaI}$ and reaches maximum value of $1.46 \times 10^{-4}$ $\mathrm{Scm}^{-1}$ was obtained for the sample containing $25 \mathrm{wt}$ $\%$ of NaI.

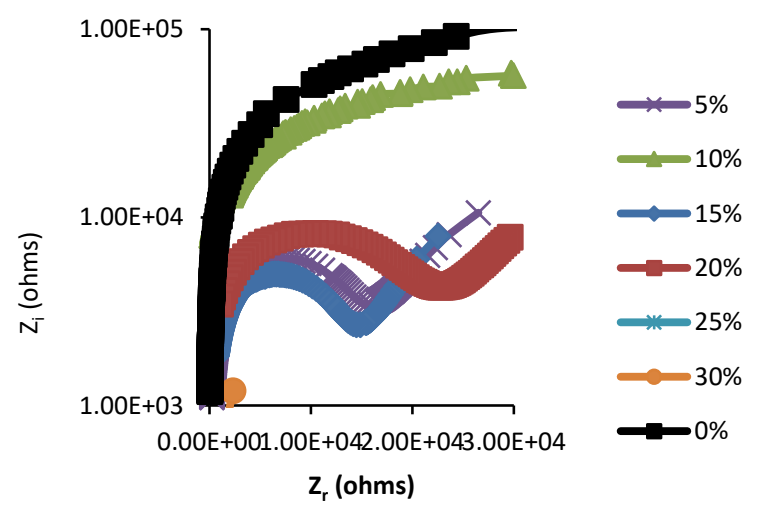

Fig. 1: Impedance plots of Cornstarch/NaI (0\%-30\%NaI, 95\% -70\% Cornstarch)

The increase of the conductivity was due to the increase in the number of the charge carrier in the sample. However, it was observed the addition of more than $25 \mathrm{wt}$ \% NaI, the conductivity electrolyte significantly decreased. This could be due to formation of ions pair producing neutral systems, thus reducing the number of free ions (Shanti, 2011).

As cornstarch is semicrystalline polymer, it has low conductance value while the addition of $\mathrm{NaI}$ increases mobile ions and the conductive system provide pathways for the movement of ions, so conductance increases (Yusof et al., 2014).

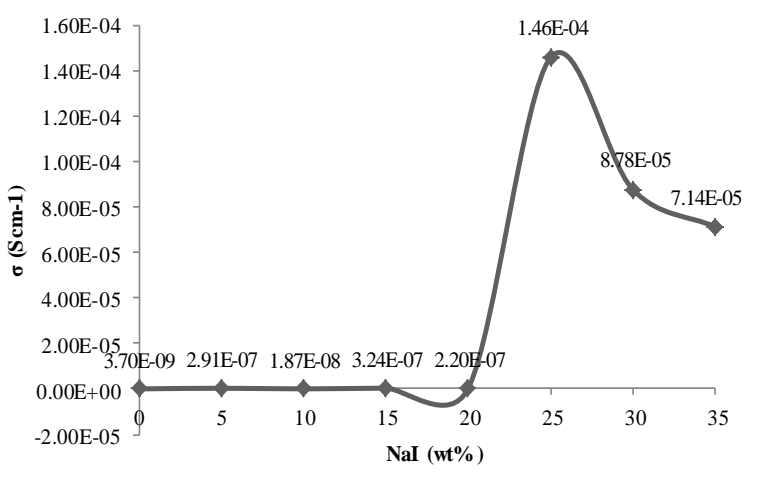

Fig. 2: Variations of electrical conductivity against percentage of $\mathrm{NaI}$ (wt. \%) at room temperature

In order to enhance the conductivity of polymer electrolyte, plasticizers, fillers and additives can been added to the solid polymer electrolytes( $\mathrm{Hu}$ et al., 2014; Ning et al., 2009). Generally, the addition of additives helps to increase the amorphous phase content of the polymer-salt complexes leading to increased mobility of the polymer chains. In this work, GO has been used to improve the conductivity 
of the biopolymer electrolyte. Fig. 3 shows the variation of conductivity of GO on biopolymer electrolyte. The highest conductivity was observed for the sample containing $10 \mathrm{wt}$. $\%$ of GO with conductivity of $2.61 \times 10^{-3} \mathrm{Scm}^{-1}$ as shown in the figure.

Interestingly, the additions $5 \mathrm{wt}$. \% of GO into the electrolyte resulted in a considerable increase in the conductivity. The increased conductivity is due to the fact that graphene provides more effective pathways for the migration of ions. The cornstarchsalt system is acting as conducting polymer material while GO plays a role as an improvement agent or filler. The morphology of GO filler has affect significantly on the formation of conductive system and the electrical properties of the composites (Das and Prusty, 2013).

GO acting as conducting enhancer dispenses itself in specified pathways in the polymer matrix. As a result, the composite with more amount of GO has lower percolation threshold and thus, conductance values increase with incorporation of GO (Diwan et al., 2012; Khan and Shakoor, 2015).

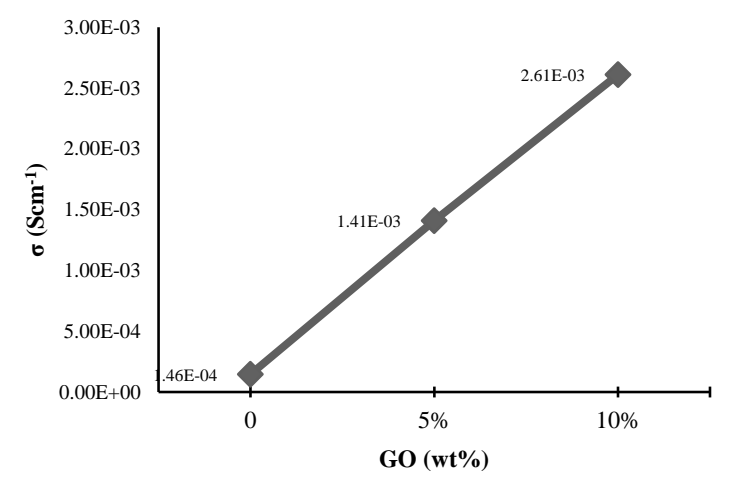

Fig. 3: Variation of conductivity as a function of wt. \% GO in Constarch/NaI

The conductivity of Cornstarch/NaI with different wt.\% of GO electrolyte against the reciprocal absolute temperature is shown in Fig. 4. The figure shows that the polymer electrolyte exhibits Arrhenius behavior due to the regression values are close to unity. The conductivity increases as a function at temperature until $343 \mathrm{~K}$ but decreases as it is heated higher than 343K.

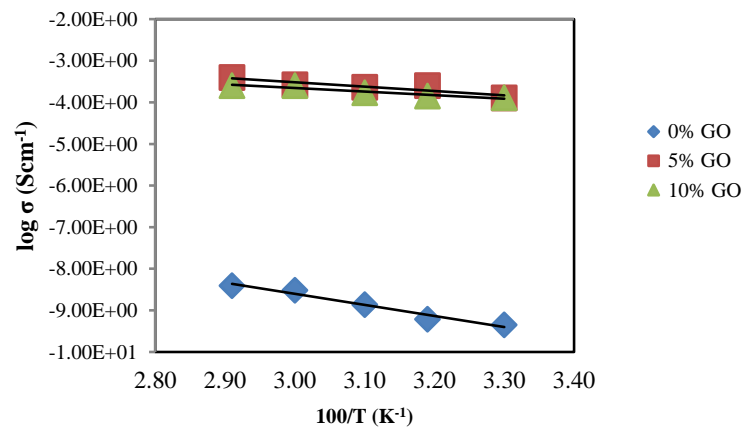

Fig. 4: Logarithmic values of conductivity of Cornstarch/NaI-GO at different temperature
Studies on the dielectric behavior give an important understanding about the trend of ionic conductivity in electrolyte system. Dielectric constant is a measurement of charge stored in a material and dielectric loss indicates the energy losses due to movement of ions when the polarity of electric field reverses rapidly (Shukur et al., 2014). Dielectric studies were done using equation (2) and (3).

$$
\begin{aligned}
\varepsilon_{r} & =\frac{z_{i}}{\omega C_{0}\left(Z_{r}^{2}+Z_{i}^{2}\right)} \\
\varepsilon_{i} & =\frac{Z_{r}}{\omega \mathrm{C}_{0}\left(\mathrm{Z}_{\mathrm{r}}^{2}+\mathrm{Z}_{\mathrm{i}}^{2}\right)}
\end{aligned}
$$

where,

$$
\begin{aligned}
& \mathrm{C}_{0}=\varepsilon_{0} \text { as permittivity in free space } \\
& \omega=2 \pi \mathrm{f}, \mathrm{f} \text { is the frequency in } \mathrm{Hz}
\end{aligned}
$$

The dielectric properties of a composite material are depend on the properties of individual phases and the mixing is controlled in such a way that individual phases are interconnected. Both the interfacial polarization ability and the homogeneous distribution of dielectric filler is the key factor to affect the dielectric performance of composites (Deshmukh and Joshi, 2015). Fig. 5 and Fig. 6 represent the variation of $\varepsilon_{\mathrm{r}}$ and $\varepsilon_{\mathrm{i}}$ with frequency for Cornstarch/NaI system at different temperature.

It is clearly shown both dielectric constant and dielectric loss increase sharply at lower frequency region indicating the accumulation of charges carrier at the electrode-electrolyte interface and the polymer electrolyte exhibit non-Debye behavior (Shanti, 2011).

The dielectric constant is very high at low frequency due to cease of ions contributions from different polarizations with the increase in the frequency (Kumar et al., 2015).

However, the dispersion of conductivity decreased at high frequency as the high periodic reversal of electric field occur so fast due to insufficient time for charge to accumulate at the interface. The polarization of mobility ion is then decreased, leading to the decrease in the values of $\varepsilon_{\mathrm{r}}$ and $\varepsilon_{\mathrm{i}}$ (Yusof et al., 2014).

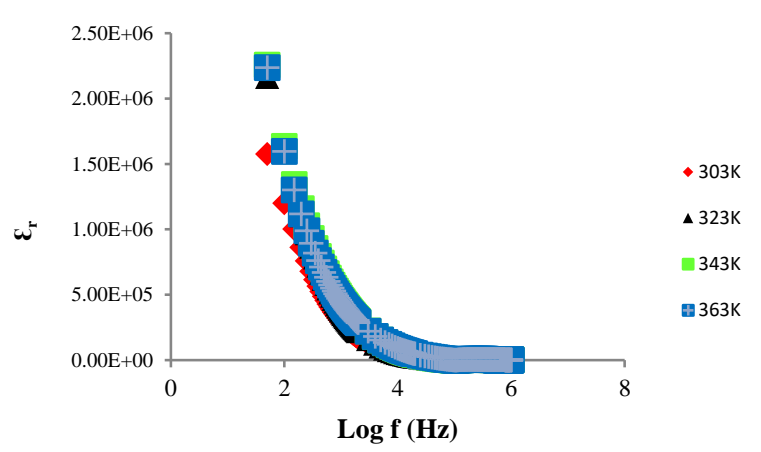

Fig. 5: Variation of real, $\varepsilon_{\mathrm{r}}$ part of dielectric constant with frequency at different temperature for $10 \% \mathrm{wt} . \% \mathrm{GO}$ in Cornstarch/NaI-GO 


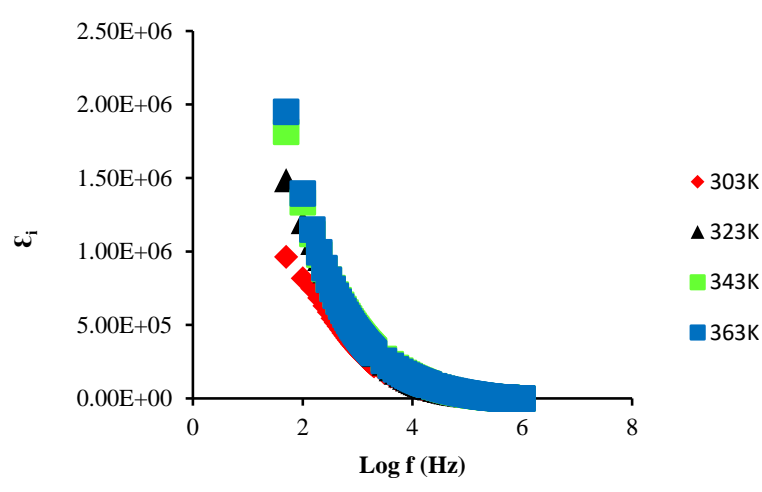

Fig. 6: Variation of imaginary, $\varepsilon_{i}$ part of dielectric constant with frequency at different temperature for $10 \mathrm{wt}$. \% GO in Cornstarch/NaI-GO

The plot of loss tangent $(\tan \delta)$ as a function of frequency shows in Fig. 7. The value of $\tan \delta$ was calculated using:

$$
\operatorname{Tan} \delta=\frac{\varepsilon_{i}}{\varepsilon_{r}}
$$

It is clearly showed that the dielectric loss $(\tan \delta)$ decreases with the frequency. The reason for this is, as the frequency increases the charge carrier cannot orient along the direction of the applied electric field, and thus the loss due to the moment of the charges is decreased. The mobile charges which belongs to polymer/salt composite namely Cornstarch/NaI and free ions which is from GO can also results in the low values of dielectric loss at higher frequencies.

It is possible to tune the dielectric constant of the material with desired value and dielectric loss can be significantly reduced, so that the material can be used for a suitable applications. Hence, Cornstarch/NaI/GO composites can be a good candidate for electrochemical devices applications.

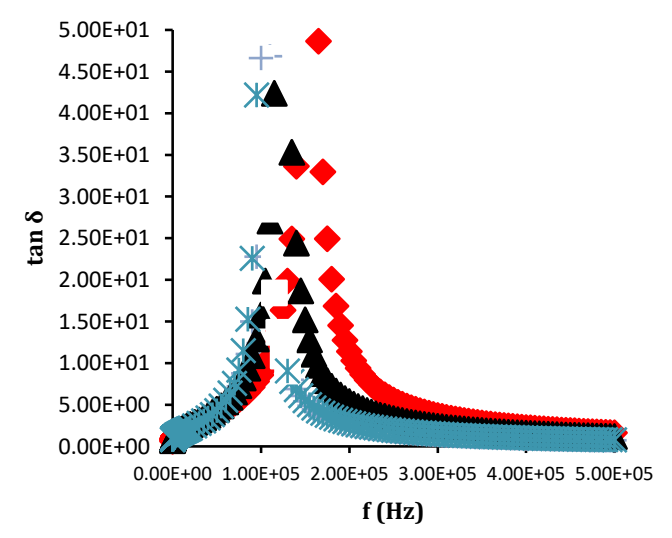

- 303K

$\Delta 323 \mathrm{~K}$

*343K

$+363 \mathrm{~K}$

Fig. 7: The frequency dependence of $\tan \delta$ of Constarch/NaI- 10 wt. \% GO at different temperature

The relaxation time $\left(t_{r}\right)$ for electrolyte was obtained from the relation,

$$
\text { trwpeak }=1
$$

where,

$\omega p e a k=$ the angular frequency of the relaxation peak.
The occurrence of relaxation time is the result of the efforts carried out by charge carriers to obey the change in the direction of the applied field. The values of $t_{r}$ for the cornstarch/NaI-10 wt. \% system at different temperature systems are shown in Table 1. From Table 1, the highest temperature of electrolyte with incorporation of $10 \mathrm{wt}$. \% GOES possesses the highest $t_{r}$ value of $1.00 \times 10^{-5} \mathrm{~s}$.

Table 1: Relaxation time for $10 \mathrm{wt} \%$ GOES in Cornstarch/NaI system

\begin{tabular}{|c|c|}
\hline Temperature $(10 \mathrm{wt} . \%$ of GO) & $\operatorname{tr}_{\mathrm{r}}(\mathrm{s})$ \\
\hline $303 \mathrm{~K}$ & $6.64 \times 10^{-6}$ \\
\hline $323 \mathrm{~K}$ & $8.33 \times 10^{-6}$ \\
\hline $343 \mathrm{~K}$ & $9.53 \times 10^{-6}$ \\
\hline $363 \mathrm{~K}$ & $1.00 \times 10^{-5}$ \\
\hline
\end{tabular}

The variation of real and imaginary part of electric modulus ( $\mathrm{M}_{\mathrm{r}}$ and $\mathrm{M}_{\mathrm{i}}$, respectively) versus frequency for the Cornstarch/NaI/GO composite is represented in Fig. 6 and 7 respectively. In Fig. 8 and 9 the height of the peaks decreases with the increasing of the temperature as relaxation time $\tau$ increases. The imaginary part of electric modulus $\left(\mathrm{M}_{\mathrm{i}}\right)$ versus frequency spectra revealed to relaxation mechanism, which recorded at high frequencies, was attributed to interfacial polarization. The frequency at the maximum of the peak represents relaxation frequency exhibit on the system (Huang et al., 2012; Latif et al., 2012)

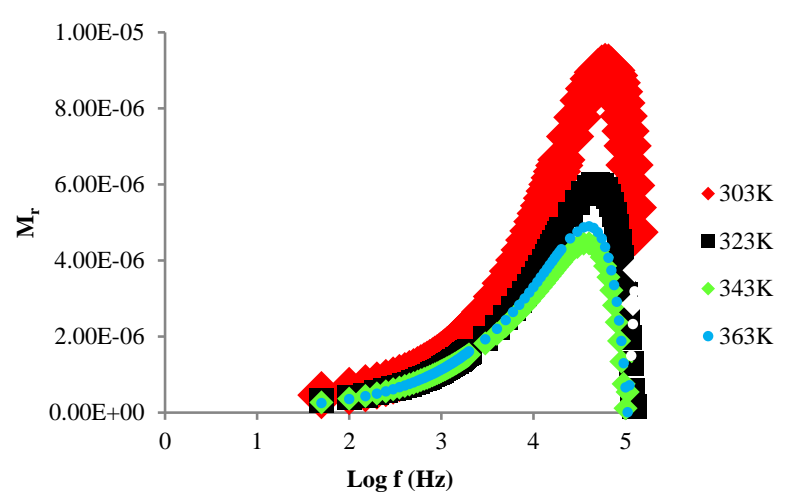

Fig. 8: Frequency dependence of real part, $\mathrm{M}_{\mathrm{r}}$ of modulus at different temperature for $10 \mathrm{wt} \% \mathrm{GO}$ in Cornstarch/NaI-GO

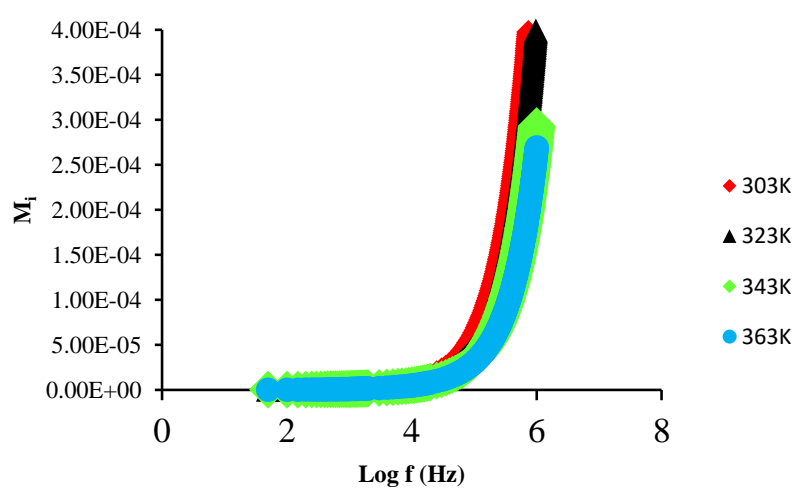

Fig. 9: Frequency dependence of imaginary part, $\mathrm{M}_{\mathrm{i}}$ of modulus at different temperature for $10 \mathrm{wt}$ \% GO in Cornstarch/NaI-GO 


\section{Conclusion}

The amount of GO on biopolymer electrolytes of cornstarch-NaI was found to have a significance enhancement on the ionic conductivity. The maximum ionic conductivity of $1.6 \times 10^{-3}$ was obtained with the sample containing of $10 \mathrm{wt}$. \% of GO. The temperature dependence of conductivity cornstarch/NaI-GO electrolytes films obeys the Arrhenius relationship. The dielectric studies of GO in electrolytes revealed the non-Debye behavior of the electrolyte. The value of $t_{r}$ is observed to increase as temperature increase shown in Table 1.

\section{References}

Balog R, Jørgensen B, Nilsson L, Andersen M, Rienks E, Bianchi M and Sljivancanin Z (2010). Bandgap opening in graphene induced by patterned hydrogen adsorption. Nature Materials, 9(4): 315-319.

Bhargav PB, Mohan VM, Sharma AK and Rao VN (2007). Structural and electrical studies of sodium iodide doped poly (vinyl alcohol) polymer electrolyte films for their application in electrochemical cells. Ionics, 13(3): 173-178.

Das TK and Prusty S (2013). Graphene-based polymer composites and their applications. Polymer-Plastics Technology and Engineering, 52(4): 319-331.

Deshmukh K and Joshi GM (2015). Embedded capacitor applications of graphene oxide reinforced poly (3, 4-ethylenedioxythiophene)tetramethacrylate (PEDOT-TMA) composites. Journal of Materials Science: Materials in Electronics, 26(8): 5896-5909.

Dikin DA, Stankovich S, Zimney EJ, Piner RD, Dommett GH, Evmenenko G and Ruoff RS (2007). Preparation and characterization of graphene oxide paper. Nature, 448(7152): 457-460.

Diwan P, Harms S, Raetzke K and Chandra A (2012). Polymer electrolyte-graphene composites: Conductivity peaks and reasons thereof. Solid State Ionics, 217: 13-18.

Dzulkurnain NA, Ahmad A and Mohamed NS (2015). $P$ (MMA-EMA) random copolymer electrolytes incorporating sodium iodide for potential application in a dye-sensitized solar cell. Polymers, 7(2):266-280.

Hu F, Li W, Zhang J and Meng W (2014). Effect of graphene oxide as a dopant on the electrochemical performance of graphene oxide/polyaniline composite. Journal of Materials Science and Technology, 30(4): 321-327.

Huang X, Zhi C, Jiang P, Golberg D, Bando Y and Tanaka $T$ (2012). Temperature-dependent electrical property transition of graphene oxide paper. Nanotechnology, 23(45): 455705, 10 pages. https: //doi.org/10.1088/0957$4484 / 23 / 45 / 455705$

Joshi GM and Deshmukh K (2016). Study of conjugated polymer/graphene oxide nanocomposites as flexible dielectric medium. Journal of Materials Science: Materials in Electronics, 27(4): 3397-3409.

Khan MS and Shakoor A (2015). Ionic conductance, thermal and morphological behavior of PEOgraphene oxide-salts composites. Journal of Chemistry, 2015: Article ID 695930, 6 pages. http://dx.doi.org/10.1155/2015/695930

Kumar KS, Pittala S, Sanyadanam S and Paik $P$ (2015). A new single/few-layered graphene oxide with a high dielectric constant of 10 6: contribution of defects and functional groups. Rsc Advances, 5(19): 14768-14779.

Kumar M, Tiwari $\mathrm{T}$ and Srivastava N (2012). Electrical transport behaviour of bio-polymer electrolyte system: Potato starch+ ammonium iodide. Carbohydrate Polymers, 88(1): 54-60.

Latif I, Alwan TB and Al-Dujaili AH (2012). Low frequency dielectric study of PAPA-PVA-GR Nano composites. Nanoscience and Nanotechnology, 2(6): 190-200.

Ning W, Xingxiang Z, Haihui L and Jianping W (2009). $\mathrm{N}$, N-dimethylacetamide/lithium chloride plasticized starch as solid biopolymer electrolytes. Carbohydrate polymers, 77(3): 607611.

Quan H, Zhang BQ, Zhao Q, Yuen RK and Li RK (2009). Facile preparation and thermal degradation studies of graphite Nano platelets (GNPs) filled thermoplastic polyurethane (TPU) Nano composites. Composites Part A: Applied Science and Manufacturing, 40(9): 1506-1513.

Shanti R (2011). Investigation on the effects of ionic liquid and ionic mixture in biodegradable polymer electrolytes. M.Sc. Thesis, Faculty of Engineering and Science, Universiti Tunku Abdul Rahman.

Shukur MF, Ithnin R and Kadir MFZ (2014). Electrical properties of proton conducting solid biopolymer electrolytes based on starch-chitosan blend. Ionics, 20(7): 977-999.

Syzdek J, Armand M, Marcinek M, Zalewska A, Żukowska G and Wieczorek W (2010). Detailed studies on the fillers modification and their influence on composite, poly (oxyethylene)-based polymeric electrolytes. Electrochimica Acta, 55(4): 1314-1322.

Yu L, Zhang Y, Tong W, Shang J, Lv F, Chu PK and Guo W (2012). Hierarchical composites of conductivity controllable polyaniline layers on the exfoliated graphite for dielectric application. Composites Part A: Applied Science and Manufacturing, 43(11): 2039-2045. 
Yusof YM, Shukur MF, Illias HA and Kadir MFZ (2014). Conductivity and electrical properties of corn starch-chitosan blend biopolymer electrolyte incorporated with ammonium iodide. Physica Scripta, 89(3): 035701, http:// stacks.iop.org/1402-4896/89/i=3/a=035701 